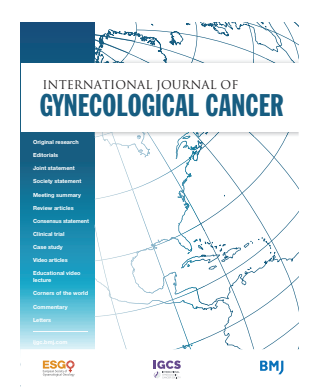

- Additional material is published online only. To view please visit the journal online (http://dx.doi.org/10.1136/ijgc2020-001718)

For numbered affiliations see end of article.

Correspondence to Dr Nicole D Fleming, Department of Gynecologic Oncology, MD Anderson Cancer Center, Houston, TX 77030, USA; nfleming@mdanderson. org

Received 4 August 2020 Revised 13 0ctober 2020 Accepted 15 October 2020 Published Online First 5 November 2020

\title{
Correlation of surgeon radiology assessment with laparoscopic disease site scoring in patients with advanced ovarian cancer
}

Nicole D Fleming (D) ," Shannon N Westin (D) , ${ }^{1}$ Larissa A Meyer (D) , ${ }^{1}$ Aaron Shafer, ${ }^{1}$ Jose Alejandro Rauh-Hain, ${ }^{1}$ Michaela Onstad, ${ }^{1}$ Lauren Cobb, ${ }^{1}$ Michael Bevers, ${ }^{1}$ Bryan M Fellman, ${ }^{2}$ Jennifer Burzawa, ${ }^{3}$ Priya Bhosale, ${ }^{4}$ Behrouz Zand, ${ }^{1}$ Amir Jazaeri, ${ }^{1}$ Charles Levenback, ${ }^{1}$ Robert L Coleman, ${ }^{1}$ Pamela T Soliman, ${ }^{1}$ Anil K Sood ${ }^{1}$

\section{HIGHLIGHTS}

- Surgeon radiology review did not correlate highly with diagnostic laparoscopy findings.

- Correlation between radiology review and laparoscopy did not vary by rank or experience.

- Surgeon radiology review best assessed stomach involvement and was worst for liver involvement.

\section{ABSTRACT}

Background Radiographic triage measures in patients with new advanced ovarian cancer have yielded inconsistent results.

Objective To determine the correlation between surgeon radiology assessment and laparoscopic scoring by disease sites in patients with newly diagnosed advanced stage ovarian cancer.

Methods Fourteen gynecologic oncology surgeons from a single institution performed a blinded review of preoperative contrast-enhanced CT imaging from patients with advanced stage ovarian cancer. Each of the patients had also undergone laparoscopic scoring assessment, between April 2013 and December 2017, to determine primary resectability using the validated Fagotti scoring method, and assigned a predictive index value score. Surgeons were asked to provide expected predictive index value scores based on their blinded review of the antecedent CT imaging. Linear mixed models were conducted to calculate the correlation between radiologic and laparoscopic score for surgeons individually, and as a group. Once the model was fit, the inter-class correlation and $95 \% \mathrm{Cl}$ were calculated.

Results Radiology review was performed on 20 patients with advanced stage ovarian cancer who underwent laparoscopic scoring assessment. Surgeon faculty rank included assistant professor $(n=5)$, associate professor $(p=4)$, and professor $(n=5)$. The kappa inter-rater agreement was $-0.017(95 \% \mathrm{Cl}-0.023$ to -0.005$)$, indicating low inter-rater agreement between radiology review and actual laparoscopic score. The inter-class correlation in this model was $0.06(0.02-0.21)$, indicating that surgeons do not score the same across all the images. When using a clinical cut-off point for the predictive index value of 8 , the probability of agreement between radiology and actual laparoscopic score was 0.56 (95\% Cl 0.49 to 0.73). Examination of disease site sub-scales showed that the probability of agreement was as follows: peritoneum 0.57 (95\% Cl 0.51 to 0.62 ), diaphragm 0.54 (95\% Cl 0.48 to 0.60$)$, mesentery $0.51(95 \% \mathrm{Cl} 0.45$ to 0.57$)$, omentum 0.61 (95\% Cl 0.55 to 0.67$)$, bowel 0.54 ( $95 \% \mathrm{Cl} 0.44$ to
0.64), stomach 0.71 (95\% Cl 0.65 to 0.76 ), and liver 0.36 (95\% $\mathrm{Cl} 0.31$ to 0.42$)$. The number of laparoscopic scoring cases, tumor reductive surgery cases, or faculty rank was not significantly associated with overall or sub-scale agreement.

Conclusions Surgeon radiology review did not correlate highly with actual laparoscopic scoring assessment findings in patients with advanced stage ovarian cancer. Our study highlights the limited accuracy of surgeon radiographic assessment to determine resectability.

\section{INTRODUCTION}

The approaches by which gynecologic oncology surgeons triage patients with advanced ovarian cancer to either primary surgery or neoadjuvant chemotherapy are diverse, inconsistent, and poorly reproducible. Pre-operative CT, serum CA-125, clinical examination, and patient factors are among the most commonly used non-invasive modalities to triage patients but have been met with variable success and it has been difficult to standardize across surgical practices. $^{1-5}$ Accurate assessment of tumor burden and pattern of spread at initial diagnosis is paramount in order to determine ability to achieve optimal tumor cytoreduction and avoid a futile laparotomy.

A laparoscopic-based scoring assessment to determine primary resectability in patients with advanced stage ovarian cancer has been previously reported in

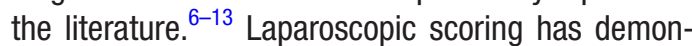
strated value in achieving an overall high positive predictive value for sub-optimal primary tumor cytoreduction, ${ }^{6-8}$ thereby reducing rates of futile laparotomy at cytoreductive surgery. ${ }^{9}$ We have previously reported that incorporating the laparoscopic scoring algorithm validated by Fagotti et al into our standard triage of patients with newly diagnosed advanced stage ovarian cancer improved our complete gross resection (R0) rates at both primary and interval 
tumor reductive surgeries. Those patients undergoing $\mathrm{R} 0$ resection at primary surgery experienced the greatest survival benefit, thus, selecting patients who would achieve the most benefit from an aggressive surgical approach. ${ }^{14}$

Some criticism of laparoscopic assessment of patients with new advanced ovarian cancer has been the need for an additional surgical procedure to obtain laparoscopic data, which could lead to delay in primary therapy, or complication risks from the laparoscopic procedure. Thus, a question is raised as to whether a thorough and systematic surgeon review of pre-operative imaging can replace laparoscopic scoring assessment. The objective of this study was to determine the correlation between surgeon radiology assessment and laparoscopic scoring by disease sites in patients with newly diagnosed advanced stage ovarian cancer.

\section{METHODS}

This retrospective imaging review protocol was approved by the University of Texas MD Anderson Cancer Center institutional review board (PA 18-0259). Fourteen gynecologic oncology surgeons from a single institution performed a blinded review of contrast-enhanced CT imaging from 20 patients with advanced stage ovarian cancer. All patients had previously undergone laparoscopic scoring assessment to determine primary resectability at tumor reductive surgery

Table 1 Clinicodemographic data from patient images reviewed

\begin{tabular}{lc}
\hline Patient characteristics & $\mathbf{n = 2 0}$ \\
\hline Age at diagnosis (years), median (range) & $65.5(36-80)$ \\
BMI $\left(\mathrm{kg} / \mathrm{m}^{2}\right)$, median (range) & $28.4(21.1-41)$ \\
ECOG performance status & \\
0 & $8(40 \%)$ \\
1 & $9(45 \%)$ \\
2 & $1(5 \%)$ \\
Unknown & $2(10 \%)$ \\
Baseline CA-125 (U/mL), median (range) & $675(61-12472)$ \\
Stage at diagnosis & \\
IIIC & $17(85 \%)$ \\
IVA & $2(10 \%)$ \\
IVB & $1(5 \%)$ \\
Laparoscopic assessment score (median) & $9(0-14)$ \\
Type of TRS & \\
Primary & $5(26 \%)$ \\
Interval & $14(74 \%)$ \\
Unknown* & 1 \\
Remaining tumor size at TRS & $15(79 \%)$ \\
R0 & $1(5 \%)$ \\
$<1$ cm & $3(16 \%)$ \\
Unknown & 1 \\
\hline
\end{tabular}

BMI, body mass index; ECOG, Eastern Cooperative Oncology Group; RO, complete gross resection; TRS, tumor reductive surgery. using the Fagotti validated scoring algorithm ${ }^{6}$ (Online supplemental table 1) between April 2013 and December 2017. These 20 patients were randomly selected from our institutional ovarian cancer database (PA 16-1010) with predictive index value scores ranging from 0 to 14 (Online supplemental table 2). The patients with predictive index value scores $<8$ were offered primary surgery and those with scores $\geq 8$ received neoadjuvant chemotherapy. Surgeons recorded predictive index value scores based on their blinded review of CT imaging and reports in the anatomic locations evaluated as a part of the Fagotti validated scoring system. Patients were excluded if CT imaging was non-contrast-enhanced or was performed outside of MD Anderson to ensure the quality of the imaging was consistent across all patient subjects.

Summary statistics of the radiologic and laparoscopic scores were calculated by the surgeon. Box plots were created to depict the scores graphically. The percent agreement (yes/no based on clinical cut-off point of 8) was calculated by surgeon and across all surgeons. The kappa inter-rater agreement statistic and corresponding $95 \% \mathrm{Cl}$ were calculated for the radiology scores. Linear mixed models were conducted to calculate the correlation between radiology and laparoscopic score for each surgeon and as a group. We regressed laparoscopic score on radiology score. A random intercept was included and our subject effect was the clinician. Once the model was fit, the inter-class correlation and $95 \% \mathrm{Cl}$ were calculated. We also fit a model with the image as our subject effect to calculate within-image correlations. We then categorized each score as agreement versus non-agreement based on the pre-defined cut-off point of 8 for treatment decision-making. Similar generalized linear mixed models were then conducted with agreement (yes/no) as outcome and clinical and demographic variables of interest as covariates to assess if certain covariates are associated with scoring agreement. Our sample size was chosen to give adequate precision for our estimates of inter-class correlation. When the sample size is 280 , a two-sided $95 \% \mathrm{Cl}$ computed using the large-sample normal approximation for an inter-class correlation based on 14 raters will extend about $0.037(0.042)$ from the observed inter-class correlation when the expected intra-class correlation is $0.7(0.3)$.

All statistical analyses were performed using Stata/MP v15.0 (College Station, Texas, USA). Study data were collected and managed using REDCap (Research Electronic Data Capture) electronic data capture tools hosted at MD Anderson. ${ }^{15}$

\section{RESULTS}

Radiology review was performed on 20 patients with advanced stage ovarian cancer who underwent laparoscopic scoring assessment. Clinical and demographic data from patients are presented in Table 1. Median age was 65.5 years (range 36-80), median body mass index was $28.4 \mathrm{~kg} / \mathrm{m}^{2}$ (range 21.1-41), and median baseline CA-125 level was $675 \mathrm{U} / \mathrm{mL}$ (range 61-12 472). The majority of patients had stage IIIC disease (85\%) and median laparoscopic predictive index value score was 9 (range $0-14$ ). Surgeon faculty academic rank included assistant professor $(n=5)$, associate professor $(n=4)$, and professor $(n=5)$. Median surgeon experience during the study period with laparoscopic scoring surgery was 13 cases (range 1-28) and tumor reductive surgery was 22.5 cases 


\section{Original research}

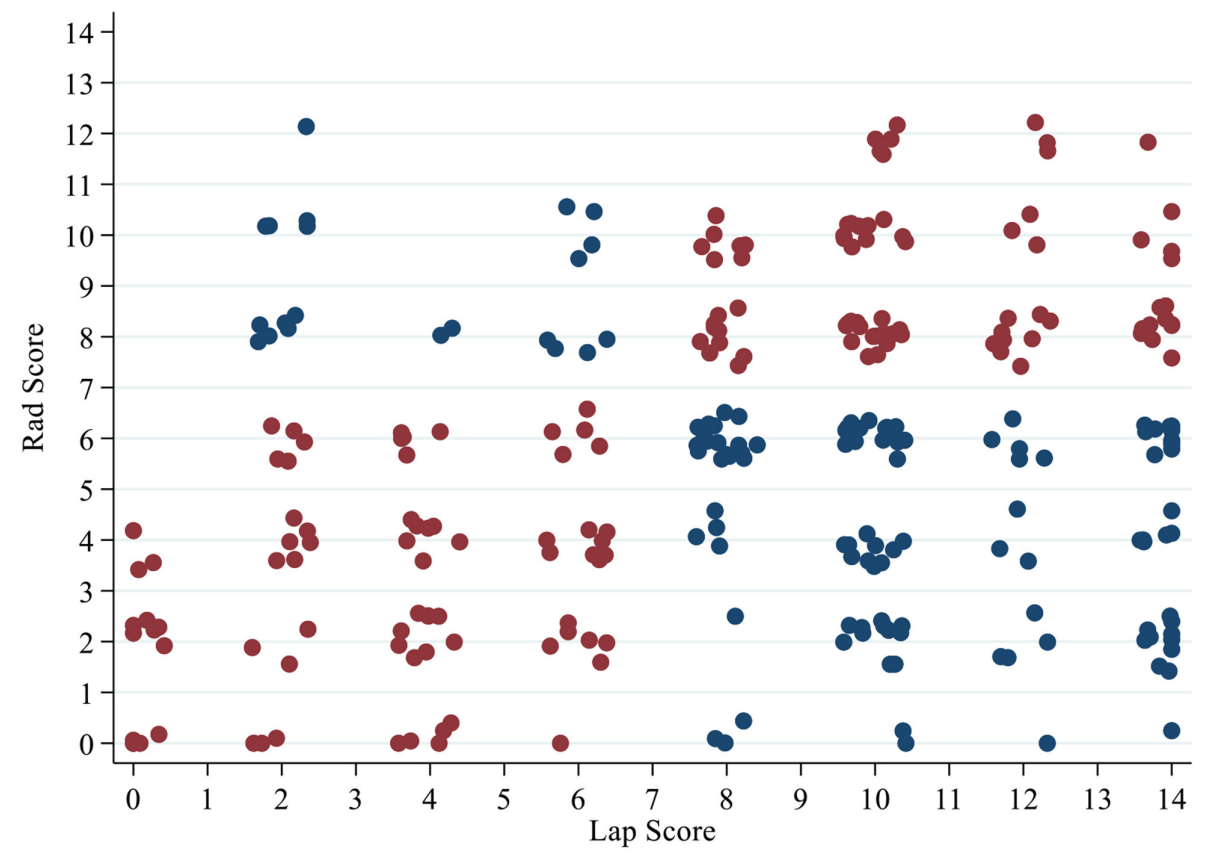

Figure 1 Scatter plot of laparoscopy compared with surgeon radiology scores. Red dots=agreement between laparoscopy score and surgeon radiology score; blue dot=no agreement between laparoscopy score and surgeon radiology score; Lap score=actual predictive index value score at the time of laparoscopy; Rad score=predictive index value score by faculty surgeon review of CT imaging.

(range 2-48). Following laparoscopic scoring assessment, five patients $(26 \%)$ underwent primary surgery and 15 patients $(74 \%)$ received neoadjuvant chemotherapy. Of those who received neoadjuvant chemotherapy, 14 patients did undergo interval tumor reductive surgery and one patient was lost to follow-up following the laparoscopic scoring assessment. The majority of patients underwent optimal tumor cytoreduction to no gross residual disease (R0, $79 \%$ ), compared with optimal $<1 \mathrm{~cm} \mathrm{(5 \% )} \mathrm{and} \mathrm{sub-optimal} \geq 1 \mathrm{~cm}$ $(16 \%)$.

Summary statistics for each surgeon's scores based on agreement of the laparoscopic assessment predictive index value score of $<8$ or $\geq 8$ are listed in Online supplemental table 3). Average agreement among all surgeons for all cases was $55.7 \%$. Agreement by experience level was $51 \%$ for assistant professor, $70 \%$ for associate professor, and $56 \%$ for professor level faculty surgeons. Figures 1 and 2 depict agreement and summary scores. The kappa inter-rater agreement was $-0.017(95 \% \mathrm{Cl}-0.023$ to -0.005$)$, indicating low inter-rater agreement between surgeon radiology review and actual laparoscopic score (Figure 2). To account for the correlations, within images and within surgeons, linear mixed models were conducted to calculate the inter-class correlation. When we treated image as the subject effect, our inter-class correlation was $0.22(95 \% \mathrm{Cl} 0.10$ to 0.40 ). An inter-class correlation of 0 would imply total agreement between surgeons about the images, thus our inter-class correlation indicates mild agreement between surgeons on scoring each image. When treating each surgeon as the subject effect, the interclass correlation in this model was $0.06(95 \% \mathrm{Cl} 0.02$ to 0.21$)$. An inter-class correlation of 1 would indicate the surgeon tended to score every image the same (ie, particularly high or low all the time). Thus our inter-class correlation in this model indicated that surgeons do not score the same across all the images.
Our next agreement analyzed was defined based on the clinical cut-off predictive index value score of 8 . Then generalized linear mixed models were conducted to assess whether surgeon academic rank, number of laparoscopic scoring procedures performed by each surgeon, and number of tumor reductive surgery cases performed by each surgeon were associated with agreement (Table 2). When using a clinical cut-off predictive index value of 8 , the probability of agreement between radiology and actual laparoscopic score was 0.56 (95\% $\mathrm{Cl} 0.49$ to 0.73$)$. The number of laparoscopic scoring cases, tumor reductive surgery cases, and surgeon title was not significantly associated with agreement.

A similar analysis was performed to assess agreement within each disease site sub-scale (Table 3) within the laparoscopic scoring algorithm. Online supplemental figure 1 includes a scatter plot of radiology and laparoscopic scores by each disease site sub-scale. Online supplemental table 4a includes the calculations of probability of agreement from the general linear models. When evaluating the disease site sub-scales, the probability of agreement was as follows: peritoneum 0.57 (95\% $\mathrm{Cl} 0.51$ to 0.62$)$, diaphragm 0.54 (95\% Cl 0.48 to 0.60$)$, mesentery 0.51 (95\% $\mathrm{Cl} 0.45$ to 0.57 ), omentum 0.61 ( $95 \% \mathrm{Cl} 0.55$ to 0.67 ), bowel 0.54 (95\% Cl 0.44 to $0.64)$, stomach 0.71 ( $95 \% \mathrm{Cl} 0.65$ to 0.76$)$, and liver $0.36(95 \% \mathrm{Cl}$ 0.31 to 0.42 ). The number of laparoscopic scoring cases, tumor reductive surgery cases, or surgeon academic rank was not significantly associated with overall or sub-scale agreement. Online supplemental table $4 \mathrm{~b}$ includes the kappa statistics for the interrater agreement on the radiology sub-scale scores.

An analysis by surgeon was performed comparing the surgeon radiology score and projected triage based on this score (primary surgery or neoadjuvant chemotherapy) with the actual laparoscopic score and actual laparoscopic triage results (Online supplemental 


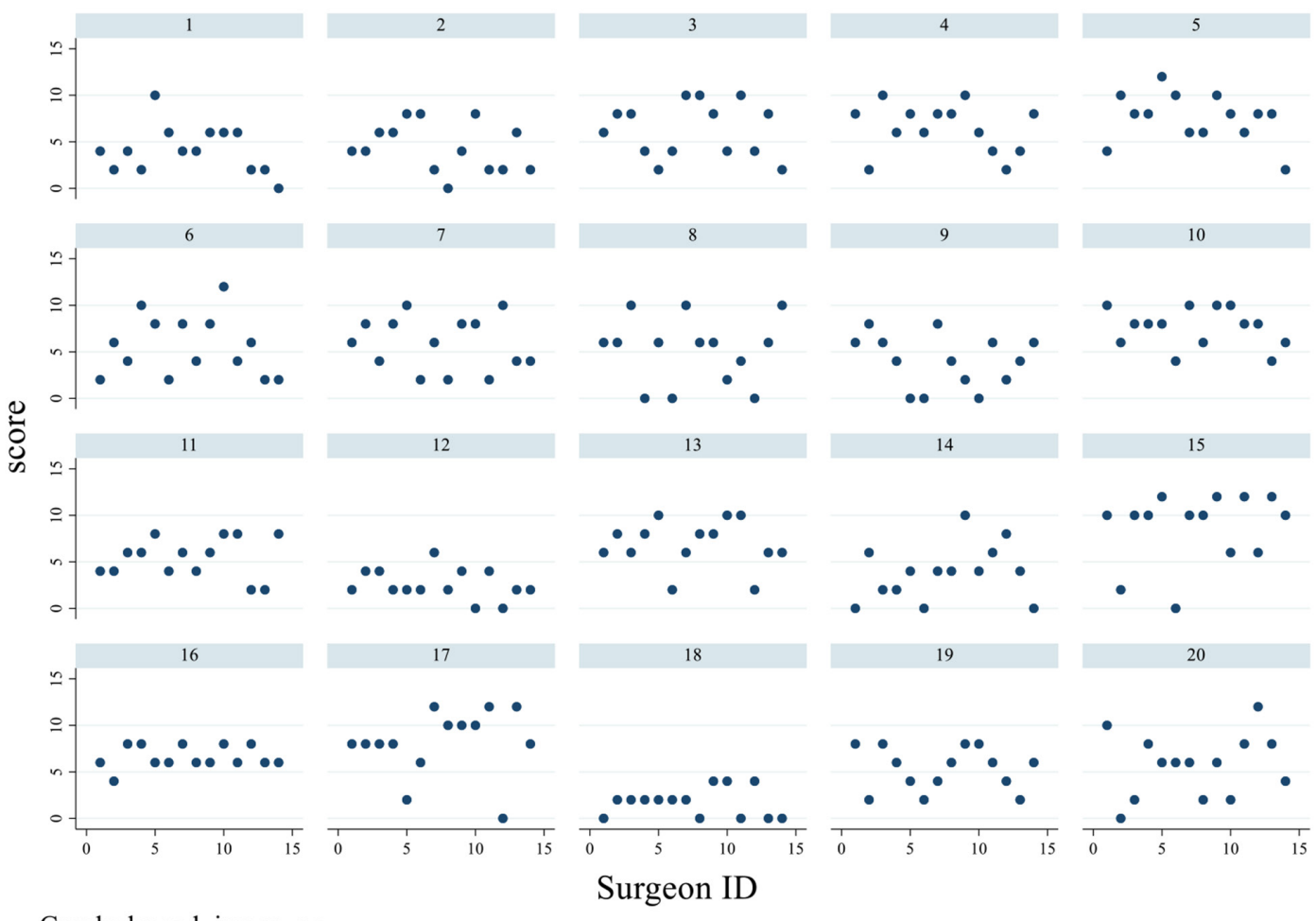

Graphs by rad_image_no

Figure 2 Scatter plot of surgeon radiology score by patient CT imaging. Blue dot represents each surgeon faculty; numbered table represents each patient CT scan reviewed; Score on $y$-axis represents predictive index value score by review of CT imaging.

table 5a-5n). Median percentage agreement in triage results (primary surgery or neoadjuvant chemotherapy) was $57.5 \%$ and median projected futile laparotomy rate was $42.5 \%$. Two patients did receive an actual laparoscopic predictive index value score of 6 , but based on disease burden assessment at the discretion of the surgeon, were triaged to neoadjuvant chemotherapy.

\section{DISCUSSION}

Surgeon radiology review did not correlate well with actual laparoscopic scoring assessment findings in patients with advanced stage ovarian cancer, regardless of surgeon experience level. By disease site sub-scale, the best agreement was noted when evaluating stomach involvement, and the worst with liver involvement. Our study highlights the limited accuracy of surgeon radiographic assessment to determine resectability.
Previous studies have shown poor correlation between CT radiographic predictors with findings at the time of tumor reductive surgery and ability to predict sub-optimal resection. ${ }^{1}$ This study, and others evaluating structured radiology reporting of disease burden by anatomic location by experienced radiologists combined with patient clinical factors, have not been reproducible to predict resection results at the time of tumor cytoreduction in advanced ovarian cancer. $^{2-4}$ Implementation of laparoscopybased scoring systems has overcome the limitations of pre-operative radiology assessment tools, ${ }^{16}{ }^{17}$ which has led to reduction in unnecessary laparotomy procedures in unresectable cases. ${ }^{7-9}$ However, widespread implementation of laparoscopic assessment for primary resectability in patients with new advanced ovarian cancer cases been met with resistance and controversy.

Our study adds to the limited literature comparing laparoscopic scoring assessment findings with detailed radiographic review

Table 2 GLMMs assessing factors associated with agreement

\begin{tabular}{lllll}
\hline Characteristics & OR & 95\% LB & 95\% UB & P value \\
\hline Scope and score number & 1.00 & 0.97 & 1.03 & 0.865 \\
TRS number & 1.00 & 0.98 & 1.01 & 0.863 \\
Title (ref: assistant) & & & & 0.113 \\
Associate professor & 2.26 & 1.05 & 4.85 & \\
Professor & 1.22 & 0.74 & 2.03 &
\end{tabular}

GLMMs, generalized linear mixed models; LB, lower bound; TRS, tumor reductive surgery; UB, upper bound. 
Table 3 Agreement of scores by disease site sub-scale

\begin{tabular}{|c|c|c|c|c|}
\hline \multirow[b]{3}{*}{ Lap score } & \multicolumn{4}{|c|}{ Radiology score } \\
\hline & \multicolumn{2}{|c|}{0} & \multicolumn{2}{|l|}{2} \\
\hline & $\mathbf{N}$ & $\%$ & $\mathbf{N}$ & $\%$ \\
\hline \multicolumn{5}{|c|}{ Peritoneal carcinomatosis } \\
\hline 0 & 54 & 51.92 & 71 & 40.57 \\
\hline 2 & 50 & 48.08 & 104 & 59.43 \\
\hline \multicolumn{5}{|c|}{ Diaphragmatic disease } \\
\hline 0 & 69 & 42.33 & 29 & 28.16 \\
\hline 2 & 94 & 57.67 & 74 & 71.84 \\
\hline \multicolumn{5}{|c|}{ Mesenteric disease } \\
\hline 0 & 57 & 41.01 & 55 & 39.01 \\
\hline 2 & 82 & 58.99 & 86 & 60.99 \\
\hline \multicolumn{5}{|c|}{ Omental site of disease } \\
\hline 0 & 69 & 64.49 & 71 & 41.04 \\
\hline 2 & 38 & 35.51 & 102 & 58.96 \\
\hline \multicolumn{5}{|c|}{ Bowel infiltration } \\
\hline 0 & 24 & 16.00 & 4 & 3.08 \\
\hline 2 & 126 & 84.00 & 126 & 96.92 \\
\hline \multicolumn{5}{|c|}{ Stomach infiltration } \\
\hline 0 & 178 & 75.11 & 18 & 62.07 \\
\hline 2 & 59 & 24.89 & 11 & 37.93 \\
\hline \multicolumn{5}{|c|}{ Liver surface } \\
\hline 0 & 67 & 28.63 & 3 & 9.38 \\
\hline 2 & 167 & 71.37 & 29 & 90.63 \\
\hline
\end{tabular}

by surgeons. There have been recent reports from Ahmed et al comparing the accuracy of CT and laparoscopy in predicting the peritoneal carcinomatosis index score. CT and laparoscopy sensitivity were $94.9 \%$, $98.3 \%$, specificity $86.7 \%, 80.4 \%$, positive predictive value $97.9 \%, 96.8 \%$, negative predictive value $72.2 \%$, $88.8 \%$, and accuracy $93.8 \%, 95.7 \%$, respectively. However, CT diagnostic performance was less accurate than laparoscopy in pelvic and small intestinal regions. The authors concluded that both CT and laparoscopy seem to be effective tools for assessment of peritoneal carcinomatosis using the peritoneal carcinomatosis index score. ${ }^{18}{ }^{19}$ No studies have compared CT diagnostic performance with laparoscopic scoring using the validated Fagotti scoring algorithm for surgical resectability. There are inherent differences in the peritoneal carcinomatosis index, which analyzes tumor size by anatomic location. ${ }^{20}$ Many previous studies have used the peritoneal carcinomatosis index to correlate surgical resectability and its impact on survival in advanced ovarian cancer. ${ }^{21-24}$ Although the peritoneal carcinomatosis index has been shown to be a validated radiologic assessment tool in advanced cancers with peritoneal spread, studies have suggested its poor usefulness as a triage test to reliably identify patients with advanced ovarian cancer who are likely to have complete cytoreductive surgery. ${ }^{23} \mathrm{~A}$ recent study suggested that selected peritoneal carcinomatosis index regions, such as the small intestine with adjacent mesentery and the hepatoduodenal ligament, are more predictive of complete resection and survival than the entire peritoneal carcinomatosis index. ${ }^{24}$ This is similar to evaluating the disease distribution in distinct anatomic areas by the Fagotti laparoscopic assessment model. However, there has been no prospective validated correlation between the peritoneal carcinomatosis index and laparoscopic assessment in advanced ovarian cancer.

The goal of any triage modality in a patient with newly diagnosed advanced ovarian cancer should be the reproducibility of the method and generalizability to practicing gynecologic oncology surgeons. Thus, the utility of structured radiology reports by specialized gynecologic oncology radiologists may not be practical in a setting that does not have access to this resource. The ability for a gynecologic oncology surgeon to interpret high-quality, contrast-enhanced CT imaging in order to make their own decisions is important. Our study, which was conducted in large tertiary cancer center, showed that our surgeons' radiology review did not correlate highly with actual laparoscopic scoring assessment findings in patients with advanced stage ovarian cancer, and thus could have altered surgical decision-making if radiology review alone had been used in these cases. Our data support the high likelihood of futile laparotomy or of a patient being inappropriately explored if radiology review alone had been used.

The strengths of our correlative study are its blinded nature and its evaluation of a large number of surgeons in a tertiary cancer center with a high volume of patients with advanced ovarian cancer. Additional strengths include evaluating surgeons of different experience and rank in their ability to predict laparoscopic score based on CT review, which leads to generalizability of the study findings to gynecologic oncology surgeons in the community. We also chose patients with varying degrees of tumor burden in order to evaluate the correlation of CT review with laparoscopy in all disease burden types. Our study is one of the first to evaluate the correlation of CT review with laparoscopy findings by disease site sub-scale based on the Fagotti algorithm. The weaknesses of the study include the small number of cases selected to review, which could lead to inherent selection bias and possible inaccuracy of the results if more cases were evaluated prospectively. We also elected not to compare the performance of a radiologist interpreting CT imaging with laparoscopy findings as laparoscopic assessment is based on surgical resectability, which is difficult to determine from a radiology perspective. Based on our results, we have included a pre-laparoscopy surgeon radiology review and scoring to our quality improvement process at our institution to provide additional prospective cases to report in the future.

In conclusion, our results suggest that the validated laparoscopic scoring algorithm should be considered the 'gold standard' assessment tool for primary resectability in patients with new advanced ovarian cancer. Further prospective studies are needed to determine the utility of novel imaging modalities which may enhance surgeon ability to predict surgical resectability, eliminating the need for laparoscopic scoring assessment.

\footnotetext{
Author affiliations

${ }^{1}$ Department of Gynecologic Oncology and Reproductive Medicine, The University of Texas MD Anderson Cancer Center, Houston, Texas, USA

${ }^{2}$ Department of Biostatistics, The University of Texas MD Anderson Cancer Center, Houston, Texas, USA

${ }^{3}$ Division of Gynecologic Oncology, Department of Obstetrics and Gynecology, University of Washington, Seattle, Washington, USA
} 
${ }^{4}$ Department of Diagnostic Imaging, The University of Texas MD Anderson Cancer Center, Houston, Texas, USA

Twitter Nicole D Fleming @nicoleflemingmd, Shannon N Westin @Shannon.Westin, Aaron Shafer @aaronshafer99, Michaela Onstad @maonstad, Robert L Coleman @rcoledude and Pamela T Soliman @PamSolimanMD

Contributors All authors contributed to writing and editing the manuscript. All authors read and approved the final manuscript.

Funding This research was in part supported by the MD Anderson Ovarian Cancer Moon Shot and by the National Institutes of Health through MD Anderson's Cancer Center support grant (P30CA016672; used the Clinical Trials Support Resource and the Biostatistics Resource Group), T32 training grant for gynecologic oncologists (T32CA101642), and Ovarian Cancer SPORE funding (CA217685), The National Institute of Health's National Cancer Institute grants K08CA234333 and K07CA201013, the Andrew Sabin Family Fellowship, the GOG Foundation scholar investigator award, the Frank McGraw Memorial Chair in Cancer Research, and the American Cancer Society research professor award.

Competing interests The authors have the following conflicts of interest to disclosure. Relevant financial activities outside the supported work; NDF: consultant/advisory board (Tesaro, BMS/Pfizer); SNW: consultant (AstraZeneca, Clovis Oncology, GSK/Tesaro, Novartis, Roche/Genentech, Eisai, Merck, Pfizer, Circulogene), research funding (ArQule, AstraZeneca, Clovis Oncology, GSK/Tesaro, Novartis, Roche/Genentech, Bayer, Cotinga Pharmaceuticals); LAM: research funding (AstraZeneca); AJ: consultant (Gerson and Lehrman Group, Guidepoint, lovance, Nuprobe, Simcere, Pact Pharma), research funding (AstraZeneca, BMS, Iovance, Aravive, Pfizer, Immatics USA, Eli Lilly); RLC: consultant (AstraZeneca, Clovis Oncology, GSK/Tesaro, Novartis, Roche/Genentech, Eisai, Merck, Pfizer, Novocure, Genmab, Gamamab, Oncosec, Tarveda), research funding (AbbVie, Genmab, Merck, AstraZeneca, Clovis Oncology, Roche/Genentech); AKS: consultant (Merck, Kiyatec), shareholder (Biopath), research funding (M-Trap). The following authors have no disclosures: PB, JAR-H, PS, AS, MO, LC, MB, BMF, JB, BZ, CL.

Patient consent for publication Not required.

Provenance and peer review Not commissioned; externally peer reviewed.

Data availability statement All data relevant to the study are included in the article or uploaded as supplementary information.

Supplemental material This content has been supplied by the author(s). It has not been vetted by BMJ Publishing Group Limited (BMJ) and may not have been peer-reviewed. Any opinions or recommendations discussed are solely those of the author(s) and are not endorsed by BMJ. BMJ disclaims all liability and responsibility arising from any reliance placed on the content. Where the content includes any translated material, BMJ does not warrant the accuracy and reliability of the translations (including but not limited to local regulations, clinical guidelines, terminology, drug names and drug dosages), and is not responsible for any error and/or omissions arising from translation and adaptation or otherwise.

\section{ORCID iDs}

Nicole D Fleming http://orcid.org/0000-0003-1785-4072

Shannon N Westin http://orcid.org/0000-0002-1922-0156

Larissa A Meyer http://orcid.org/0000-0002-2687-7463

\section{REFERENCES}

1 Axtell $\mathrm{AE}$, Lee $\mathrm{MH}$, Bristow RE, et al. Multi-Institutional reciprocal validation study of computed tomography predictors of suboptimal primary cytoreduction in patients with advanced ovarian cancer. $J$ Clin Oncol 2007;25:384-9.

2 Suidan RS, Ramirez PT, Sarasohn DM, et al. A multicenter prospective trial evaluating the ability of preoperative computed tomography scan and serum CA-125 to predict suboptimal cytoreduction at primary debulking surgery for advanced ovarian, fallopian tube, and peritoneal cancer. Gynecol Oncol 2014;134:455-61.

3 Suidan RS, Ramirez PT, Sarasohn DM, et al. A multicenter assessment of the ability of preoperative computed tomography scan and CA-125 to predict gross residual disease at primary debulking for advanced epithelial ovarian cancer. Gynecol Oncol 2017:145:27-31.

4 Ferrandina G, Sallustio G, Fagotti A, et al. Role of CT scan-based and clinical evaluation in the preoperative prediction of optimal cytoreduction in advanced ovarian cancer: a prospective trial. $\mathrm{Br} J$ Cancer 2009;101:1066-73.

5 Aletti GD, Eisenhauer EL, Santillan A, et al. Identification of patient groups at highest risk from traditional approach to ovarian cancer treatment. Gynecol Oncol 2011;120:23-8.

6 Fagotti A, Ferrandina G, Fanfani F, et al. A laparoscopy-based score to predict surgical outcome in patients with advanced ovarian carcinoma: a pilot study. Ann Surg Oncol 2006;13:1156-61.

7 Fagotti A, Ferrandina G, Fanfani F, et al. Prospective validation of a laparoscopic predictive model for optimal cytoreduction in advanced ovarian carcinoma. Am J Obstet Gynecol 2008;199:642.e1-642.e6.

8 Fagotti A, Vizzielli G, De laco P, et al. A multicentric trial (OlympiaMITO 13) on the accuracy of laparoscopy to assess peritoneal spread in ovarian cancer. Am J Obstet Gynecol 2013;209:462. e1-462.e11.

9 Rutten MJ, van Meurs HS, van de Vrie R, et al. Laparoscopy to predict the result of primary cytoreductive surgery in patients with advanced ovarian cancer: a randomized controlled trial. J Clin Oncol 2017;35:613-21.

10 Fagotti A, Vizzielli G, Fanfani F, et al. Introduction of staging laparoscopy in the management of advanced epithelial ovarian, tubal and peritoneal cancer: impact on prognosis in a single institution experience. Gynecol Oncol 2013;131:341-6.

11 Fagotti A, Vizzielli G, Costantini B, et al. Learning curve and pitfalls of a laparoscopic score to describe peritoneal carcinosis in advanced ovarian cancer. Acta Obstet Gynecol Scand 2011;90:1126-31.

12 Brun J-L, Rouzier R, Uzan S, et al. External validation of a laparoscopic-based score to evaluate resectability of advanced ovarian cancers: clues for a simplified score. Gynecol Oncol 2008;110:354-9.

13 Petrillo M, Vizzielli G, Fanfani F, et al. Definition of a dynamic laparoscopic model for the prediction of incomplete cytoreduction in advanced epithelial ovarian cancer: proof of a concept. Gynecol Oncol 2015;139:5-9.

14 Fleming ND, Nick AM, Coleman RL, et al. Laparoscopic surgical algorithm to triage the timing of tumor reductive surgery in advanced ovarian cancer. Obstet Gynecol 2018;132:545-54.

15 Harris PA, Taylor R, Thielke R, et al. Research electronic data capture (REDCap)--a metadata-driven methodology and workflow process for providing translational research informatics support. J Biomed Inform 2009;42:377-81.

16 Hansen JM, Sood AK, Coleman RL, et al. Concordance of a laparoscopic scoring algorithm with primary surgery findings in advanced stage ovarian cancer. Gynecol Oncol 2018;151:428-32.

17 van de Vrie R, Rutten MJ, Asseler JD, et al. Laparoscopy for diagnosing resectability of disease in women with advanced ovarian cancer. Cochrane Database Syst Rev 2019;3.

18 Abdalla Ahmed S, Abou-Taleb H, Ali N, et al. Accuracy of radiologiclaparoscopic peritoneal carcinomatosis categorization in the prediction of surgical outcome. Br J Radiol 2019;92:20190163.

19 Ahmed SA, Abou-Taleb H, Yehia A, et al. The accuracy of multidetector computed tomography and laparoscopy in the prediction of peritoneal carcinomatosis index score in primary ovarian cancer Acad Radiol 2019;26:1650-8.

20 Jacquet $\mathrm{P}$, Sugarbaker $\mathrm{PH}$. Clinical research methodologies in diagnosis and staging of patients with peritoneal carcinomatosis. Cancer Treat Res 1996:82:359-74.

21 Schmidt S, Meuli RA, Achtari C, et al. Peritoneal carcinomatosis in primary ovarian cancer staging: comparison between MDCT, MRI, and 18F-FDG PET/CT. Clin Nucl Med 2015;40:371-7.

22 Mazzei MA, Khader L, Cirigliano A, et al. Accuracy of MDCT in the preoperative definition of peritoneal cancer index $(\mathrm{PCl})$ in patients with advanced ovarian cancer who underwent peritonectomy and hyperthermic intraperitoneal chemotherapy (HIPEC). Abdom Imaging 2013;38:1422-30.

23 Avesani G, Arshad M, Lu H, et al. Radiological assessment of peritoneal cancer index on preoperative CT in ovarian cancer is related to surgical outcome and survival. Radiol Med 2020;125:770-6.

24 Rosendahl M, Harter P, Bjørn SF, et al. Specific regions, rather than the entire peritoneal Carcinosis index, are predictive of complete resection and survival in advanced epithelial ovarian cancer. Int $J$ Gynecol Cancer 2018;28:316-22. 